\title{
Hemibalismo-hemicoreia em estado hiperglicêmico não cetótico: distúrbio do movimento associado ao diabetes melito
}

\author{
Hemiballism-hemichorea with non-ketotic hyperglycemia: \\ movement disorder related to diabetes mellitus
}

Viviane Flumignan Zétola' ${ }^{1}$ Bruno Verschoor', Fernando M. Lima', Francisco E. Ottmann', Eloisa Doubrawa', Eduardo Paiva', Maurício de Carvalho', Hélio A. G. Teive²

\begin{abstract}
SUMÁRIO
O diabetes melito, especialmente quando descompensado, pode culminar em várias complicações neurológicas, sendo o desenvolvimento de movimentos involuntários uma das formas mais raras. $O$ estado hiperglicêmico não cetótico em pacientes idosos, que se apresentam com movimentos tipo balismo-coreia associados a alterações nos exames de imagem cerebral (tomografia computadorizada e/ou ressonância magnética), constitui uma síndrome de caracterização recente e de poucos relatos na literatura. Apresentamos o caso de um paciente admitido com história de movimentos involuntários do tipo hemibalismo-hemicoreia à esquerda associado a estado hiperglicêmico com hemoglobina glicada de 14,4\%. 0 exame tomográfico de crânio revelou área hiperdensa em topografia de gânglios da base à direita. Após controle glicêmico adequado, houve melhora progressiva e recuperação do quadro neurológico, com desaparecimento completo da lesão hiperdensa inicial. Arq Bras Endocrinol Metab. 2010;54(3):335-8
\end{abstract}

\section{SUMMARY}

Diabetes mellitus, especially when not under control, can lead to several neurological complications being the development of involuntary movements one of the rarest presentations. Nonketotic hyperglycemia in aged patients who present with ballismus-chorea movements and cerebral image alterations in computerized tomography (CT) and magnetic resonance constitute a syndrome of recent characterization and few cases in literature. We present a case of a 75 year-old male patient admitted with history of hemiballismus-hemichorea movements, hyperglycemia, glycated hemoglobin of $14.4 \%$ and CT with a hyperdense area in the topography of the right basal ganglia. After glycemic control, the neurological signs resolved completely and the initial hyperdense lesion disappeared. Arq Bras Endocrinol Metab. 2010;54(3):335-8
Departamento de Clínica Médica, Hospital de Clínicas, Universidade Federal do Paraná (HC-UFPR), Curitiba, PR, Brasil ${ }^{2}$ Serviço de Neurologia, HCUFPR, Curitiba, PR, Brasil
$\mathrm{B}$ alismo e coreia são distúrbios do movimento hipercinético diferenciados pela amplitude e distribuição de grupos musculares, evidentes no repouso ou na ação, e que tendem a desaparecer durante o sono (1). Acometem mais frequentemente um lado do corpo, sendo assim denominados hemibalismo ou hemicoreia.

Geralmente resultam de lesões estruturais no núcleo subtalâmico e estriado contralateral, podendo ser de várias etiologias, principalmente secundários a eventos vasculares (2). Na ausência de lesão vascular focal, alterações metabólicas, neoplasia cerebral e infecções do sistema nervoso central (SNC) devem ser consideradas (3).

Existem raros relatos na literatura. Estima-se que a incidência de hemibalismo seja 1 caso para 500 mil na população geral (2).

$\mathrm{O}$ estado hiperosmolar hiperglicêmico $(\mathrm{EHH})$ representa uma das complicações mais graves do diabetes melito (DM), mais frequentemente observado em dia- 
béticos tipo 2 com idade acima de 65 anos. $\mathrm{O}$ quadro pode se manifestar com diversas alterações neurológicas, como alterações no sensório, convulsões, hemiplegia transitória e movimentos involuntários (4).

\section{RELATO DE CASO}

Paciente masculino, 75 anos, hipertenso e dislipidêmico. Recente diagnóstico de DM com diversos internamentos por descompensação. Em uso irregular de metformina $850 \mathrm{mg}$ ao dia e sinvastatina $20 \mathrm{mg}$ ao dia.

Há dois meses iniciou quadro insidioso e progressivo de confusão mental, parestesias e movimentos involuntários de grande amplitude em dimídio esquerdo, predomínio proximal. Relato de atendimento em Unidade primária de Saúde (US) com glicemia de $600 \mathrm{mg} / \mathrm{dL}$ e manejo com "soro" com melhora parcial do quadro.

$\mathrm{Na}$ admissão, persistia a queixa de confusão mental (escore de minimental: 8/30) e movimentos involuntários contínuos de menor amplitude em mão e pé esquerdos, tipo coreia. Exame neurológico com discreta hemiparesia esquerda (grau IV + simétrico) e movimentos coreiformes em dimídio esquerdo. Monitoração de glicemia capilar variando ao redor de $300-400 \mathrm{mg} / \mathrm{dL}$, hemoglobina glicada ( $\mathrm{HbAlc}$ ) de 14,4\%, osmolaridade sérica calculada de $301 \mathrm{mOsm} / \mathrm{kg}$. Análise do liquor sem alterações. Ausência de outras alterações metabólicas.

Realizada tomografia de crânio (TC), que demonstrou lesão hiperdensa, heterogênea, medindo cerca de $2,7 \times 2,0 \mathrm{~cm}$, sem efeito expansivo ou retrátil, na topografia do núcleo lentiforme direito, sugerindo pseudocalcificações (Figura 1). O paciente permaneceu internado para controle glicêmico mais rigoroso, tendo atenuado os movimentos até remissão completa no período de 12 dias. Houve melhora parcial do quadro confusional. Nova imagem de crânio 15 dias após o exame inicial mostrou desaparecimento tomográfico completo da lesão inicial (Figura 2).

Após 60 dias da alta, o paciente foi readmitido com quadro semelhante de movimentos involuntários tipo coreia, acometendo mesmo dimídio corporal. Apresentava história de ingesta abusiva de bebida alcoólica antes do reinício dos movimentos. Durante a internação, não foi constatado nenhum outro distúrbio metabólico. O exame neurológico não encontrou novos déficits e o quadro cognitivo permanecia inalterado. Mantinha um bom controle glicêmico com uso de metformina $2.550 \mathrm{mg} / \mathrm{d}$ e glibenclamida $10 \mathrm{mg} / \mathrm{d}$, tendo nova dosagem de $\mathrm{HbAlc} \mathrm{em} \mathrm{7,8 \% .} \mathrm{Foi} \mathrm{realizada} \mathrm{ressonância}$ magnética (RM), que demonstrou lesão hiperintensa em Tl (sequelar) e infartos lacunares contralaterais (Figuras 3 e 4). Em razão da recorrência dos movimentos mesmo sob controle glicêmico, optou-se por iniciar haloperidol $5 \mathrm{mg} / \mathrm{d}$, visando a melhor controle do quadro de hemibalismo-hemicoreia. Recebeu alta com melhora parcial dos movimentos e manutenção do tratamento do DM.

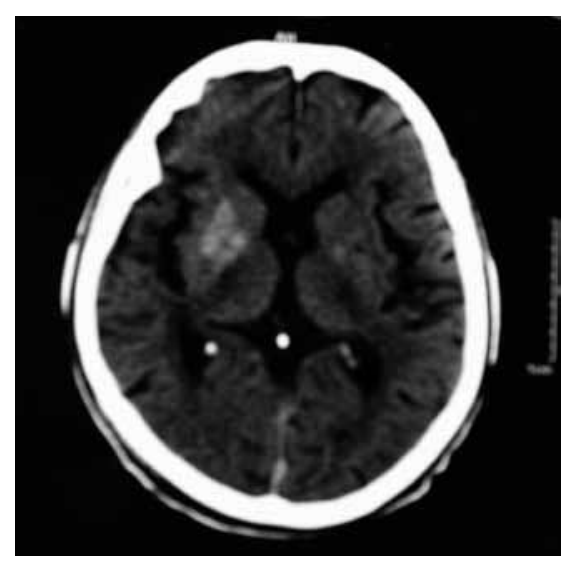

Figura 1. Tomografia computadorizada de crânio realizada na admissão.

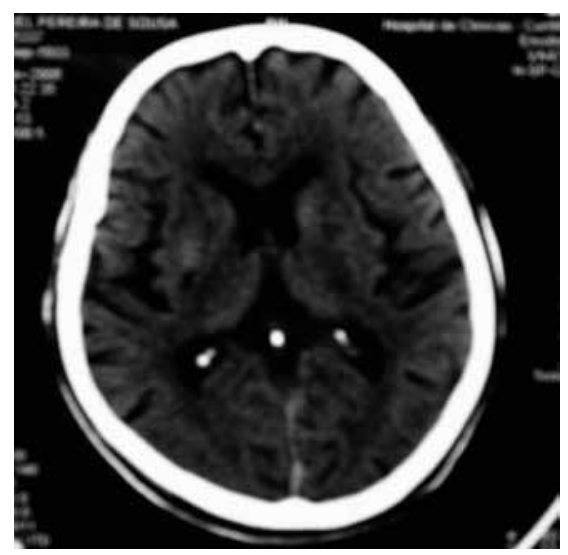

Figura 2. Tomografia computadorizada de controle realizada 15 dias após.

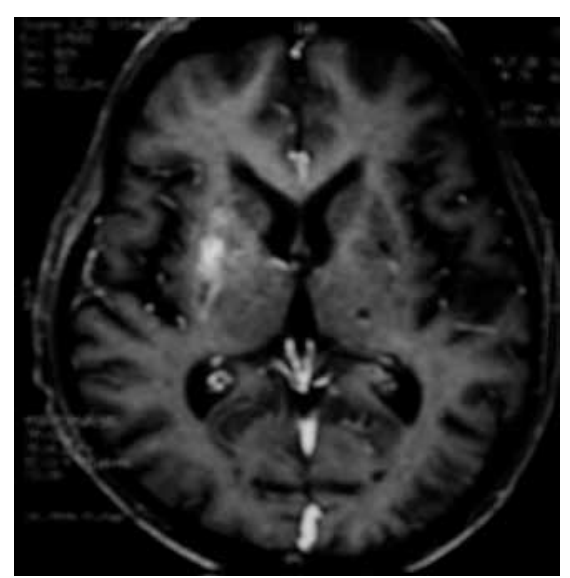

Figura 3. Ressonância magnética T1 - corte axial (reinternação). 


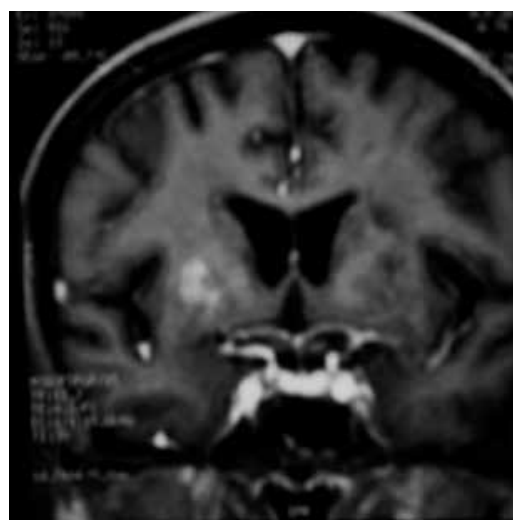

Figura 4. Ressonância magnética T1 - corte coronal (reinternação).

\section{DISCUSSÃO}

A combinação de hemicoreia-hemibalismo, hiperglicemia não cetótica e envolvimento dos gânglios da base em exames de imagem tem sido considerada como uma síndrome única. Ocorre geralmente como complicação de diabetes tipo 2 de longa duração, mas também tem sido descrita como manifestação inicial do $\operatorname{DM}(5,6)$.

Uma revisão sistemática de 53 casos publicados encontrou uma predisposição em idosos (idade média 71,1) e mulheres $(56,6 \%)$. Na admissão, estes apresentavam altos níveis de glicose sérica (média 481 mg/dl) e HbAlc (média $14,4 \%$ ). Hemicoreia foi a apresentação inicial em $88,6 \%$ dos casos e $73 \%$ obtiveram recuperação completa (7).

Exames de imagem descrevem mais frequentemente hiperdensidade (TC) ou hiperintensidade (ressonância magnética em $\mathrm{Tl}$ ) no território do ramo lateral estriatal da artéria cerebral média (8). Os principais diagnósticos diferenciais dessas lesões nos gânglios da base são calcificações e hemorragia. A diferenciação pode ser feita pela evolução clínica e por características imagenológicas (3).

Lin e cols. (8) propuseram alguns critérios para a caracterização dessa síndrome: 1) movimentos coreiformes ou balísticos em pelo menos dois dos seguintes: face unilateral, pescoço, membro superior ou membro inferior; 2) grande elevação da glicemia; 3 ) lesão hiperintensa no estriado contralateral na TC ou RM; 4) desaparecimento abrupto da discinesia após controle glicêmico; 5) TC ou RM sem evidências de acidente vascular cerebral (AVC), infecção ou lesões inflamatórias; 6) sem evidências de outra alteração metabólica, uso de drogas ou história de doença degenerativa.

As alterações cerebrais que ocorrem durante o estado hiperglicêmico não cetótico ainda não são totalmente entendidas. Postula-se que o estado hiperglicêmico provoque uma mudança para o metabolismo anaeróbio e leve à diminuição secundária do GABA (ácido gama-aminobutírico), uma vez que este funciona como substrato energético alternativo durante a anaerobiose, contribuindo para a disfunção dos gânglios da base (9). Nos casos cetóticos, o GABA pode ser ressintetizado, explicando assim a falta de associação entre coreiabalismo e cetoacidose diabética (4).

A gênese dos movimentos balísticos pode estar relacionada a uma redução na atividade palidal pela lesão nos núcleos subtalâmico, caudado e putâmen, com consequente perda na inibição talâmica e uma superativação da via direta (7).

Lesões cerebrovasculares prévias podem levar à disfunção da barreira hematoencefálica e, em sinergismo com os efeitos metabólicos da hiperglicemia, facilitar o extravasamento de hemácias por diapedese e formação de hemorragia petequial nos gânglios da base (10-13). Estudo por tomografia por emissão de prótons (PET) sugere falha no metabolismo de glicose local, o que está de acordo com essas hipóteses e reforça a hiperglicemia como fator desencadeador (14).

A análise histopatológica dessas lesões é conflitante, mostrando áreas de perda neuronal, gliose e astrocitose reativa (8). Mestre e cols. (10) encontraram hemorragia focal no estriado e sugeriram que as alterações vistas nos exames de imagem correspondem inicialmente a sangue e posteriormente a depósitos de hemossiderina.

Embora nosso paciente não tenha sido submetido inicialmente à RM ou a um exame funcional, a melhora clínica e radiológica após o controle hiperglicêmico corrobora fortemente as alterações como secundárias a distúrbio metabólico. A recorrência pode ocorrer em até $17 \%$ dos casos, principalmente em função de um novo quadro de hiperglicemia ou uso irregular de medicamentos para controle dos sintomas (7).

Esse caso demonstra uma manifestação clínico-radiológica única do DM e enfatiza os benefícios do reconhecimento precoce e tratamento efetivo. O prognóstico é bom, com o controle dos níveis glicêmicos sendo muitas vezes suficiente para a melhora do quadro. Nos casos em que os movimentos persistem apesar da normalização da glicose, medicações (incluindo neurolépticos, benzodiazepínicos e antiepiléticos) podem ser úteis $(5,15)$.

Declaração: os autores declaram não haver conflitos de interesse científico neste estudo.

\section{REFERÊNCIAS}

1. Block H, Scozzafava J, Ahmed SN, Kalra S. Uncontrollable movements in patient with diabetes mellitus. CMAJ. 2006;175:871. 
2. Coral P, Teive HAG, Werneck LC. Hemibalismo: relato de 8 casos. Arq Neuropsiquiatr. 2000;58(3-A):698-703.

3. Chang MH, Chiang HT, Lai PH, Sy CG, Le SJ, Lo YU. Putaminal petechial hemorrhage as the cause of chorea: a neuroimaging study. J Neurol Neurosurg Psychiatry. 1997;63:300-3.

4. Lin JJ, Chang MK. Hemiballism-hemichorea and non-ketotic hyperglycemia. J Neurol Neurosurg Psychiatry. 1994;57:748-50.

5. Kranick, SM, Price RS, Prasad S, Hurtig HI. Clinical reasoning: a 52-year-old woman with subacute hemichorea. Neurology. 2008;71:e59-62.

6. Yasuhara A, Wada J, Makino H. Bilateral dystonia in type 1 diabetes: a case report. J Med Case Reports. 2008;2:352.

7. Oh SH, Lee KY, Im JH, Lee MS. Chorea associated with non-ketotic hyperglycemia and hyperintensity basal ganglia lesion on T1weighted brain MRI study: a meta-analysis of 53 cases including 4 present cases. J Neurol Sci. 2002;20:57-62.

8. Lin JJ, Lin GY, Shih C, Shen WC. Presentation of striatal hyperintensity on T1-weighted MRI in patients with hemiballism-hemichorea caused by non-ketotic hyperglycemia: report of seven new cases and a review of literature. J Neurol. 2001;248:750-5.
9. Guisado R, Arieff Al. Neurological manifestations of diabetic comas: correlation with biochemical alterations in the brain. Metabolism. 1975;24:665-79.

10. Mestre AT, Ferreira JJ, Pimentel J. Putaminal petechial haemorrhage as the cause of non-ketotic hyperglycaemic chorea: a neuropathological case correlated with MRI findings. J Neurol Neurosurg Psychiatry. 2007;78:549-50.

11. Shan DE. Hemichorea-hemiballism associated with hyperintense putamen on T1-weighted MR images: an update and a hypothesis. Acta Neurol Taiwan. 2004;13:170-7.

12. Felicio AC, Chang CV, Godeiro-Junior C, Okoshi MP, Ferraz HB. Hemichorea-hemiballism as the first presentation of type 2 diabetes mellitus. Arq Neuropsiquiatr. 2008;66(2-A):249-50.

13. Lai PH,Tien RD, Chang MH, Teng MM, Yang CF, Pan HB, et al. Chorea-ballismus with nonketotic hyperglycemia in primary diabetes mellitus. Am J Neuroradiol. 1996;17:1057-64.

14. Hsu JL, Wang HC, Hsu WC. Hyperglycemia-induced unilateral basal ganglion lesions with and without hemichorea. A PET study. J Neurol. 2004;251:1486-90.

15. Sitburana O, Ondo WG. Tetrabenazine for hyperglycemic-induced hemichorea-hemiballismus. Mov Disorder. 2006;21(11):2023-5. 